\title{
Measurement of the exercising blood flow during rhythmical muscle contractions assessed by Doppler ultrasound: Methodological considerations
}

\author{
Takuya Osada $^{1,2,3}$, Bengt Saltin ${ }^{3}$, Stefan P. Mortensen ${ }^{3}$, Göran Rådegran ${ }^{3,4}$ \\ ${ }^{1}$ Department of Sports Medicine for Health Promotion, Tokyo Medical University, Tokyo, Japan \\ ${ }^{2}$ Cardiac Rehabilitation Center, Tokyo Medical University Hospital, Tokyo, Japan \\ ${ }^{3}$ The Copenhagen Muscle Research Centre, Rigshospitalet, University of Copenhagen, Copenhagen, Denmark \\ ${ }^{4}$ The Clinic for Heart Failure and Valvular Disease, Skåne University Hospital, and Department of Cardiology, IKVL, Lund University, \\ Lund, Sweden \\ Email: DENTACMAC@aol.com
}

Received 16 October 2012; revised 22 November 2012; accepted 29 November 2012

\section{ABSTRACT}

Given the recent technological developments, ultrasound Doppler can provide valuable measurements of arterial blood flow with high temporal resolution. In a clinical setting, measurements of hemodynamics is used to monitor, diagnose and manage changes in blood velocity profile for cardiac valve disease, relatively large vessel stenosis and other cardiovascular diseases. In health science and preventive medicine for cardiovascular disease with exercise therapy, evaluation of cardiac and vascular function is a useful indicator not only at rest but also during exercise, leading to improved exercise tolerance as well as physical activity. During exercise, the increase in oxygen uptake (calculated as product of arterial blood flow to the exercising limb and the arterio-venous oxygen difference) is directly proportional to the work performed. The increased oxygen demand is met through a central mechanism, an increase in cardiac output and blood pressure, as well as a peripheral mechanism, an increase in vascular conductance and oxygen extraction (major part in the whole exercising muscles) from the blood. Therefore, the determination of the local blood flow dynamics (potential oxygen supply) feeding to rhythmic muscle contractions can contribute to the understanding of the factors limiting the work capacity including, for instance the muscle metabolism, substance utilization and vasodilatation in the exercising muscle. Using noninvasive measures of pulsed Doppler ultrasound the validity of evaluating blood velocity/flow in the forearm or lower limb conduit artery feeding to the muscle is demonstrated during rhythmic muscle exercise; however the exercising blood velocity profile (fast Fourier transformation) due to muscle contractions is always seen as a physiological variability or fluctuations in the magnitude in blood velocity due to the spontaneous muscle contraction and relaxation induced changes in force curve intensity. Considering the above mentioned variation in blood velocity in relation to muscle contractions may provide valuable information for evaluating the blood flow dynamics during exercise. This review presents the methodological concept that underlines the methodological considerations for determining the exercising blood velocity/flow in the limb conduit artery in relation the exercise model of dynamic leg exercise assessed by pulsed Doppler ultrasonography.

Keywords: Exercising Blood Flow; Doppler Ultrasound; Muscle Contraction; Physiological Flow Variations

\section{INTRODUCTION}

Given technological developments within the last decades, ultrasound Doppler can provide valuable measurements of arterial blood flow with high temporal resolution in the cardiovascular system. The determination of blood velocity in the feeding conduit artery at rest and during rhythmic muscle contractions during the exercise has an impact on the transient changes in hemodynamics [1-3]. The investigation of the blood flow supply due to continuous muscle contractions may require the evaluation of the effect of physical activity on regulation among central and peripheral hemodynamics.

The oxygen transport via blood flow to the working muscles is crucial for the exercise capacity. Furthermore, the magnitude of blood flow in the exercising muscle may also be related to the blood volume of redistribution via systemic circulation as seen in previous studies focused on cardiovascular regulations in human $[4,5]$. 
The oxygen uptake is theoretically evaluated by the product of cardiac output and arterio-venous oxygen concentration difference, and consequently peripheral conduit arterial blood flow in the working muscle is one indicator for the metabolic demand in the local large muscle groups [6]. Moreover, to detect utilization in the leg requires comprehensive leg blood flow and arteriovenous substance concentration difference [7,8].

Peripheral circulatory changes during exercise correspond to the stress imposed on the cardiovascular system $[3,9]$. Cardiac output increases with increasing exercise intensity along with enhanced skeletal muscle vasodilatation and muscle pumping in the exercising muscle. As the perfusion in the active muscle is furthermore one indicator of oxygen delivery to the muscles, blood velocity and flow in the feeding conduit arteries to working skeletal muscle may also give us valuable information regarding the hemodynamic response to the exercise (particularly for the large muscle groups in the upper or lower limb), for instance, cardiac function in cardiovascular disease cases, disuse muscle atrophy syndrome, clinical targeting in rehabilitation and other research in biomedical science. The advantages of Doppler ultrasound with high temporal resolution in evaluating blood velocity are as mentioned above, however, the measurement of valid blood velocity value should be done carefully to account for the influence of changes in the voluntary muscle contraction force in inducing blood velocity fluctuations. The blood velocity oscillates at rest as an effect of the heart beat and blood pressure, and during repeated muscle contractions of exercise these oscillations are even more pronounced as they are also influenced by the intramuscular pressure variations. Acknowledging the variability in the conduit arterial blood velocity feeding into voluntary rhythmic muscle contractions is valuable information for precise determination of exercising blood flow under various muscle contraction intensities and frequencies.

Therefore, the purpose of the present review is to summarize the methodological considerations for determining the exercising blood velocity/flow in the limb conduit artery during thigh muscle kicking exercise (dynamic knee extensor exercise model) assessed by Doppler ultrasound. The structure of this paper is organized as follows: 1) Validation of exercising blood flow; 2) Physiological variations in blood velocity during rhythmic muscle contractions and 3) Evaluation of the netexercising blood flow between muscle contraction cycle and cardiac beat-by-beat cycle.

\section{METHODOLOGICAL CONSIDERATION}

\subsection{Participants}

The data in this review are from participants as follows: total number of participants, 50 healthy males; age range: 21 - 36 years; height range: $174-193 \mathrm{~cm}$; and body weight range: 59 - $97 \mathrm{~kg}$. Participants had no previous history of cardiovascular disease, gastrointestinal disease, hypertension, or anaemia, and no abnormality of the peripheral vasculature. The studies were conducted according to the principles of the Declaration of Helsinki (1976) and with the approval of the Institutional Ethics Committee of the author's institution. All participants gave their written consent and were informed of the nature and purpose of the study, as well as potential risks and discomfort. The participants also understood that they could withdraw from the study at any time without consequence.

\subsection{Exercise Model}

Determinations of blood flow to contractile muscles are the most important focus of the present review. Precise and stable measurements in conduit arteries assessed by Doppler ultrasound should be sustained during exercise. Whole body exercise methods such as walking and running on a treadmill do not easily allow measurement of upper-and lower-limb blood flow using Doppler ultrasound in these models as motion artifacts are present. There is also difficulty in fixing the ultrasound Doppler probe. Whole lower limb muscle blood flow may be measured using the one-legged, repeated kicking (dynamic knee-extensor) exercise model described by Andersen and Saltin [10]. In this exercise model, the subject performs leg kicking whereas the leg is passively returned by the cycle ergometer. Consequently, the work is confined to the quadriceps muscle group and the model allows stable measurements of femoral arterial blood velocity using Doppler ultrasound because the subject is seated (Figure 1). Therefore, all hemodynamic data described in this review are from one-legged repeated kicking (dynamic knee-extensor) exercise with the activation of the large thigh muscle group.

\subsection{Hemodynamic Measurements}

Ultrasound instrumentation: The measurements were performed using a Doppler ultrasound instrument (Model CFM 800, Vingmed Sound, Horten, Norway) equipped with an annular phased array transducer (Vingmed Sound) probe (11.5-mm diameter). The imaging frequency was $7.5 \mathrm{MHz}$ and the Doppler frequencies varied between 4.0 and $6.0 \mathrm{MHz}$ (high-pulsed repetition frequency mode, 4 - $36 \mathrm{kHz}$ ). Blood velocity was measured with the probe at the lowest possible insonation angle and always $<60^{\circ}$ [11]. The mean value of the insonation angle was $\sim 50^{\circ}$, which remained constant throughout the experiments for each individual. The probe position was stable and the sample volume was 


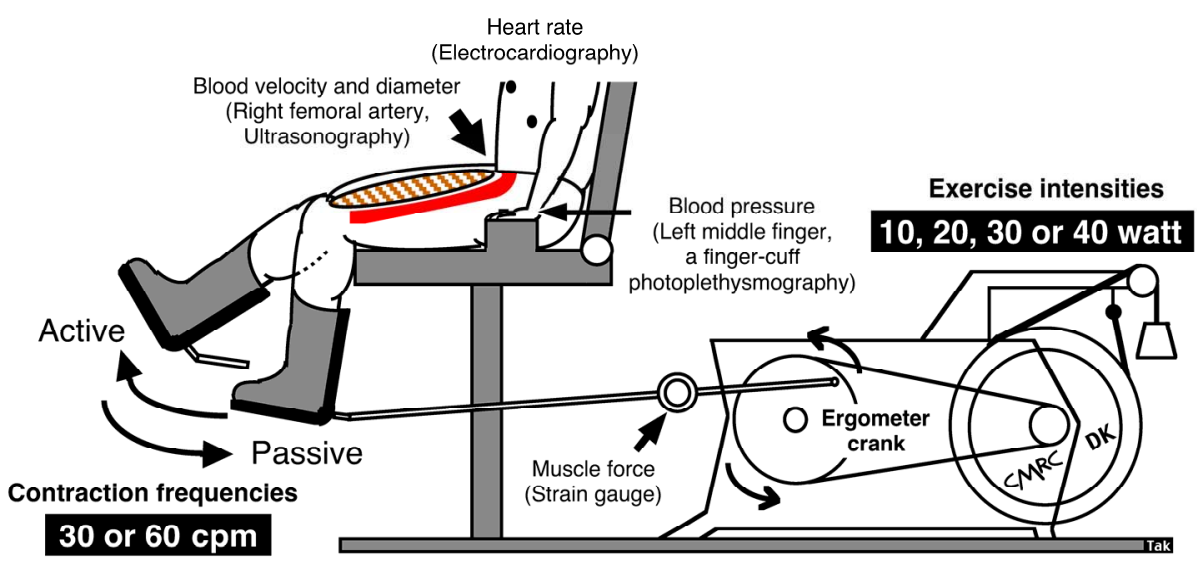

Figure 1. One-legged dynamic kicking (knee extensor) exercise as the model of rhythmic thigh muscle contractions.

precisely positioned in the center of the vessel and adjusted to cover the diameter width of the vessel.

Blood Velocity, Vessel Diameter and Blood Flow: The measurements of blood velocity and blood flow in the femoral artery using Doppler ultrasound has previously been validated and shown to produce accurate absolute values both at rest and during leg exercise such as rhythmical thigh muscle contractions [1-3,12,13]. Compared with thermodilution, the high temporal resolution of pulsed Doppler ultrasound additionally enables continuous measurement of blood velocity throughout the knee-extensor exercise [1-3,9,14,15].

The angle-corrected, time and space-averaged, and amplitude-weighted mean blood velocities were measured. Mean blood velocity was defined by averaging the mean blood velocity trace including both negative and positive values [3,9]. The blood velocity parameter was measured in relation to the blood pressure curve. The site of blood velocity and vessel diameter measurements in the femoral artery was distal to the inguinal ligament but above the bifurcation into the branch of the superficial and deep femoral artery. This location minimizes turbulence from the femoral bifurcation and the influence of blood flow from the inguinal region. In addition, the arterial diameter is not affected by the contractions and relaxations at this site located proximal to the muscle. The blood velocity measurements were performed when steady-state had been reached after 3 min of one-legged, dynamic knee extensor, as previously described $[3,13,14]$. The systolic and diastolic diameters of the femoral artery were measured on a monitor relative to the electromyography at rest. The mean vessel diameter was calculated in relation to the temporal duration of the blood pressure curve as; [(systolic vessel diameter value $\times 1 / 3)+$ (diastolic vessel diameter value $\times 2 / 3$ )] [3]. The diameters were measured under perpendicular insonation at rest before exercise. The value of the vessel diameter at rest (pre-exercise) was used to calculate femoral arterial blood flow during rest and during one-legged, dynamic knee extensor, since the diameter does not vary between rest and steady-state exercise [3,4,12,16-18]. Steady-state one-legged blood flow was calculated by multiplying the cross-sectional area $\left[\right.$ Area $\left.=\pi \times(\text { vessel diameter } / 2)^{2}\right]$ of the femoral artery, with the angle corrected, time and space-averaged, and amplitude (signal intensity)-weighted mean blood velocity, where blood flow $=$ mean blood velocity $\times$ cross-sectional area. Thus, the changes in blood flow dynamics were basically parallel to the changes in blood velocity.

Muscle force and work rate: Kicking muscle force was measured using a strain gauge. Variations in muscle force were taken to represent oscillations in intramuscular pressure, as these parameters have been shown to temporally correlate closely to each other during dynamic knee extensor $[3,14,19]$. The external workload (work rate) was calculated according to the knee extensor ergometer model $[10,20]$, defined as: external workload $($ watt $)=[$ contraction frequency $($ contractions per minute, $\mathrm{cpm}) / 60 \mathrm{~s}] \times[$ distance of one knee extensor revolution $(6 \mathrm{~m})] \times\left[\operatorname{load}(\mathrm{kg}) \times 9.81\left(\mathrm{~m} / \mathrm{s}^{2}\right)\right]$. The specific loads applied were $0.333,0.667,1.0$ and $1.333 \mathrm{~kg}$ at 10 , 20,30 and 40 watt, respectively, at $30 \mathrm{cpm}$; and 0.083 , $0.167,0.333,0.5,0.667,0.833$ and $1.0 \mathrm{~kg}$ at $5,10,20,30$, 40,50 and 60 watt at $60 \mathrm{cpm}$.

The external workload was evaluated by integrating delta $\mathrm{dP}$ during the muscle contraction phase, where $\mathrm{dP}$ (to time integral) $=\mathrm{dF}[\mathrm{N}] \mathrm{i} \times \mathrm{R} \times \operatorname{Sin}[$ alpha] $\mathrm{i} \times$ revolution per minute/60, were determined for each knee extensor kicking session. The external workload $=$ integral of $\mathrm{dP}$ from time integral $=0($ where alpha $=0)$ to time integral $=$ $\mathrm{x}$ (where alpha $=\mathrm{pi}$ ); $\mathrm{dF}[\mathrm{N}] \mathrm{i}$, force (in Newtons) on the kicking arm transducer to time integral; $\mathrm{R}$, Length of pedal arm in meters; Sin[alpha]i, Sin to horizontal angle to time integral; revolution per minute, actual angular velocity in rounds per minute to time integral; and $\mathrm{dF}[\mathrm{N}] \mathrm{i} \times$ $\mathrm{R} \times \operatorname{Sin}[a l p h a] \mathrm{i}$ " is the delta torque $[\mathrm{Nm}]$ to time integral. 
The achieved workload determined by this method was displayed in real time on a monitor, visible for the subjects, to maintain the target workload during dynamic knee extensor.

\section{EVALUATION OF EXERCISING BLOOD FLOW}

\subsection{Validation of Exercising Blood Flow during Rhythmic Muscle Contractions}

In previous reports, peripheral hemodynamic measurements have been performed using the thermodilution technique for leg blood flow during dynamic kneeextensor exercise [10]. However, this invasive technique has the limitation of poor time resolution of blood flow. Several other techniques have previously been developed that enable estimates to be made of arterial inflow, venous outflow, and local blood flow within a muscle [2127]. Whereas many of the techniques are impaired by different methodological limitations, the indicator thermodilution and the ultrasound Doppler method have both been found to give repeatable measurements of the same magnitude during both rest and dynamic knee extensor exercise [3,28] (Figure 2).

There is a positive linear relationship between leg blood flow in femoral artery and target work rate $(10,20$,

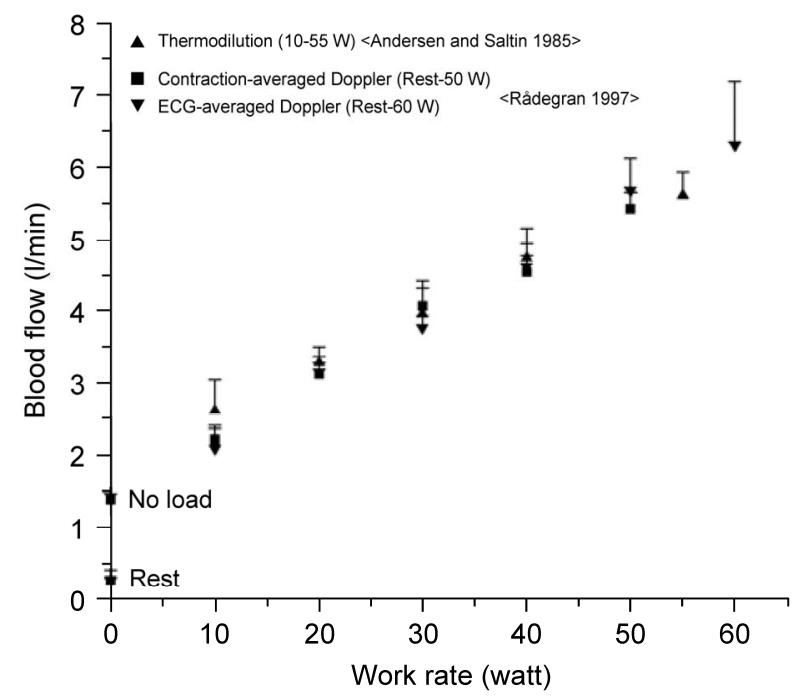

Figure 2. Blood flow during incremental one-legged dynamic kicking (knee extensor) exercise at 60 contractions per minute, estimated from mean blood velocity and aver- aged on a beat-by-beat basis or continuously measured and analyzed in relation to muscle contraction cycle. The blood flow increases linearly with incremental target exercise in- tensities of work rate during steady-state exercise. This im- plies that an enhanced vasodilatation is elicited, in relation to the increased average muscle force exerted at high work rates, to meet the elevated metabolic activity. Figure adap- ted from Rådegran [3], reproduced with permission from The American Physiological Society.
$30,40,50$ and 60 watt) in relation to rhythmic thigh muscle contractions at 60 contractions per minute (Figure 2). With the rapid increase in energy requirements during exercise, equally rapid circulatory adjustments are essential in order to meet the increase need for oxygen and nutrients by the exercising muscle. In addition, thermodilution blood flow measurements obtained under similar experimental conditions by Andersen and Saltin [10] are closely related to those obtained by Doppler ultrasound. Thus, blood flow measured by Doppler ultrasound is valid not only at rest but also during incremental one-legged dynamic knee extensor exercise. The precision and accuracy of the Doppler technique has been improved by sampling the blood velocity (muscle contraction cycle) continuously instead of averaging the velocity in relation to each cardiac cycle (beat-by-beat cycle) (see $\leftrightarrow$ in Figure 3).

The Doppler technique can be used to differentiate between physiological and methodological variations in flow, as well as detect rapid changes in flow induced by exercise (dynamic or static), different metabolic states (muscle contraction intensity or frequency), or any other type of vasodilatation such as the reperfusion period after arterial occlusion or infusion of a vasodilator substance.

\subsection{Physiological Variations in Blood Velocity during Rhythmic Muscle Contractions}

The continuous recordings can clearly determine the magnitude of the physiological variability in blood velocity by the contraction-relaxation-induced variations in muscle force, and consequently the intramuscular pressure variations, along with the superimposed influence of the blood pressure as well as the tonic influence of the state of vasodilatation $[3,13,29]$. The high intramuscular pressure during muscle contractions may consequently temporarily reduce or even reverse the blood velocity, depending on the relationship between the intramuscularand arterial blood pressure. The major extent of the blood velocity and flow consequently occurs during the muscle relaxation phase $[3,13,30]$. Blood velocity fluctuated in relation to the state of vasodilatation and the muscle contraction-relaxation duty cycles, indicated by the oscillations in muscle force.

In generally, the blood velocity increased to its highest value at the systolic blood pressure phase during muscle relaxation, and significantly decreased to its lowest value at the diastolic blood pressure phase during muscle contraction (Figure 3). The blood velocity showed an intermediate value at the systolic blood pressure phase during muscle contraction and at the diastolic blood pressure phase during muscle relaxation, respectively. The blood velocity curve was furthermore retrograde in the diastolic blood pressure phase during muscle contraction. 


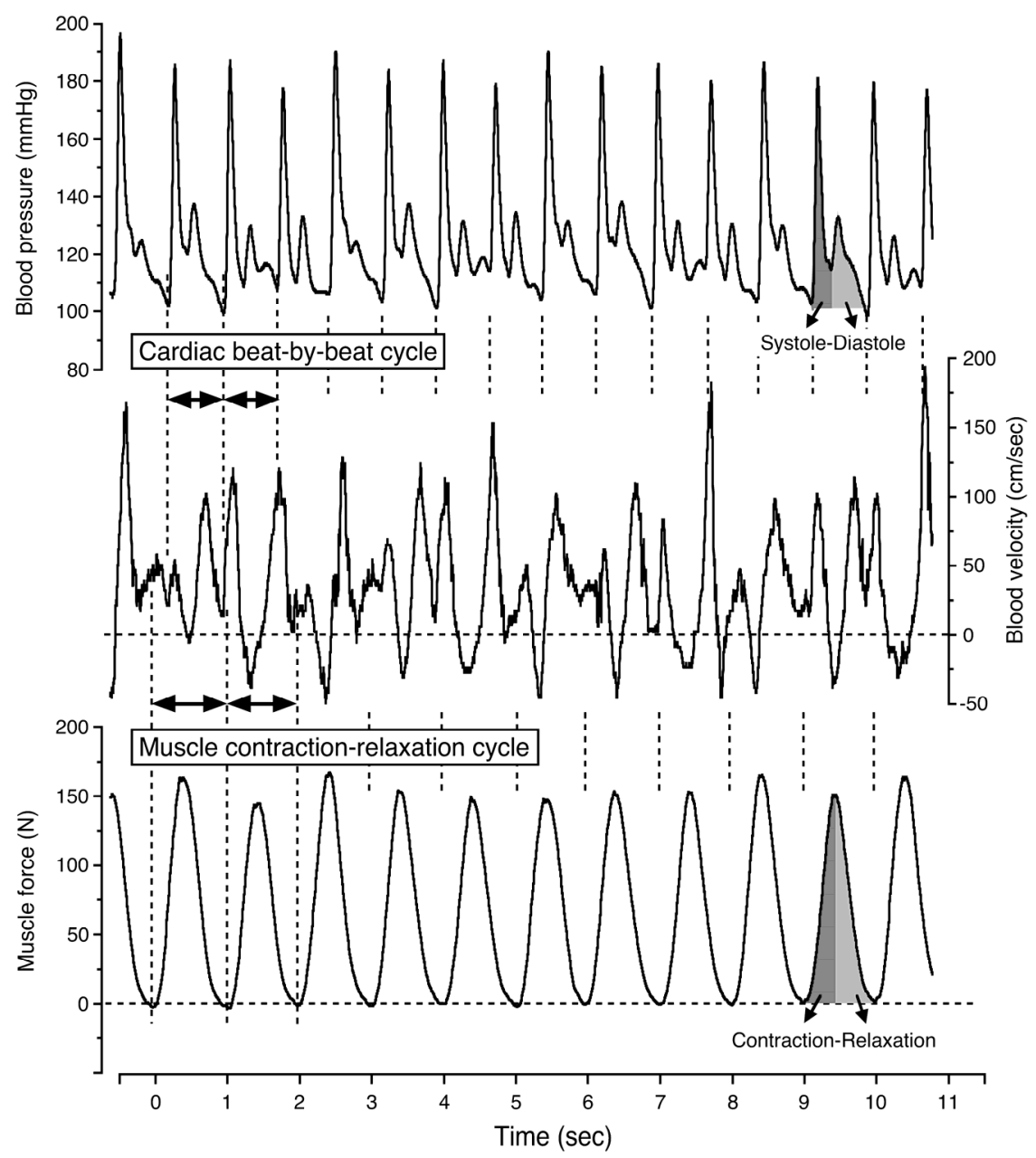

Figure 3. Continuous recording of blood velocity, blood pressure and muscle force during steady-state one-legged dynamic kicking (knee extensor) exercise at 20 watt and 60 contractions per minute. Figure adapted from Osada and Rådegran [29], reproduced with permission from Edizioni Minerva Medica.

In Figure 4 the limited view of blood velocity profile in relation to single muscle contraction-relaxation and single cardio systolic-diastolic beat, the blood velocities during the systolic and diastolic phases were found continuously in parallel with the blood pressure curve during the muscle contraction and muscle relaxation phases determined from the electromyography and the muscle force curve.

Four variations in the coupling between the blood pressure curve and the state of muscle contraction and relaxation were indicated; the systolic phase during muscle contraction, the diastolic phase during muscle contraction, the systolic phase during muscle relaxation, and the diastolic phase during muscle relaxation. The formation of the blood velocity profile and flow was influenced by the intramuscular pressure, as indicated by the muscle force curve, and the superimposed influence of the blood pressure in relation to the systolic and diastolic phases. The magnitude in blood flow value measured by the 4 variations indicates the large difference in work rates during the muscle relaxation phase and those of the muscle contraction phase at systolic and diastolic points, respectively (Figure 5).

The difference in the blood velocity values due to normal physiological variations raises the question as to how much sampling duration is necessary to determine the acceptable range of valid net-blood velocity value during steady-state rhythmic repeated voluntary muscle contraction exercise.

\subsection{Muscle Contraction-Induced Blood Velocity Variability and Net-Blood Velocity Value Evaluated by Sampling Number of Muscle Contraction-Relaxation Cycle}

A physiological variation in femoral artery is observed due to each muscle contraction during steady-state, onelegged, repeated kicking (dynamic, knee-extensor) exercise as mentioned above in Section 3.2. This was predo- 


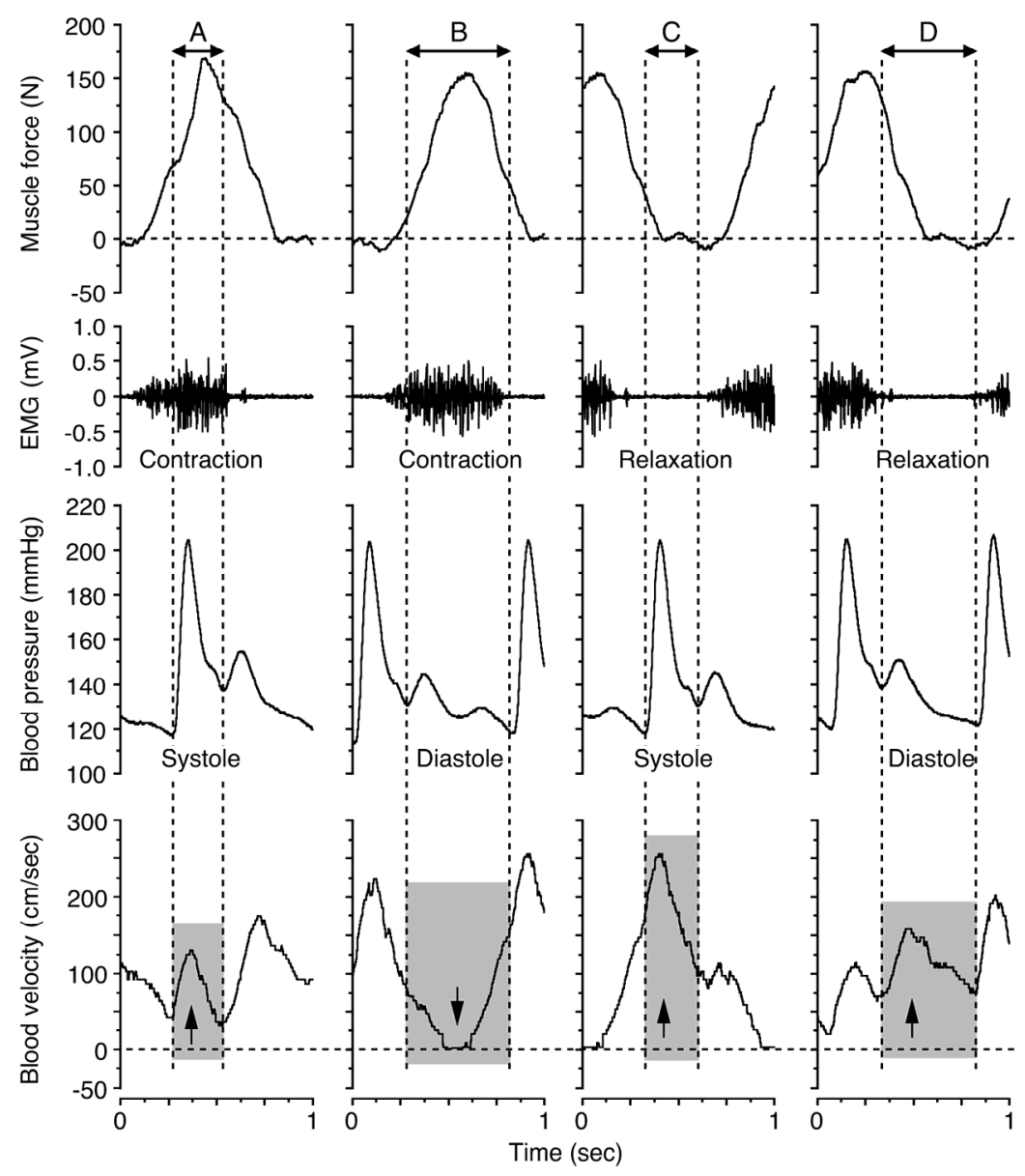

Figure 4. Blood velocity profile for the systolic and diastolic phases during the muscle contraction and muscle relaxation phases at 20 watt and 60 contractions per minute. The arrows down and up indicate the influence on the blood velocity, de- pending on the magnitude of, and temporal relation between, the muscle force ( $\approx$ intramuscular pressure) and the blood pressure, respectively. Figure adapted from Osada and Rådegran [30], reproduced with permission from The Physiological Society of Japan.

minantly related to the muscle contraction-induced oscillations in intramuscular pressure and the influence of the superimposed waves on the cardiac cycle and arterial perfusion pressure.

During steady-state exercise with the rhythmic muscle contractions at $60 \mathrm{cpm}(0.5 \mathrm{~s}$ muscle contraction $-0.5 \mathrm{~s}$ muscle relaxation), it was found that the blood flow variability was smaller from 15 -s $\sim$ to $\sim 60$-s (longer periods) than shorter periods, such as $3-\mathrm{s}$, as determined by averaging blood flow measurements over time periods [3]. This difference in blood flow variability may depend on the sampling duration which may potentially include the various magnitudes in fragments of exercising blood velocity profile (see $\leftrightarrow$ in Figures 3 and 4 ).

The magnitude of the muscle contraction-relaxationinduced physiological variability in blood velocity between "consecutive muscle contraction cycle" during steady-state rhythmic thigh muscle contractions at diff- erent contraction intensities (10,20,30 and 40 watt) and contraction frequencies ( 30 and $60 \mathrm{cpm}$ ) is examined (see simultaneous recording of hemodynamics parameters at 20 watt and $60 \mathrm{cpm}$ in Figure 6). The steadystate suggests the condition of less variability of voluntary muscle contraction force, blood pressure and heart rate.

Measurement with the methodological concept for steady-state net-blood flow value requires determining whether the muscle contraction-relaxation cycle induced flow variability will be reduced by sampling more muscle contraction-relaxation cycles. In addition, it was important to determine the sampling duration of the muscle contraction-relaxation cycle that had the smallest variability required to obtain accurate steady-state netblood flow measurements at rest and during one-legged, repeated kicking (dynamic knee extensor) exercise. The blood flow variability was determined for seven con- 


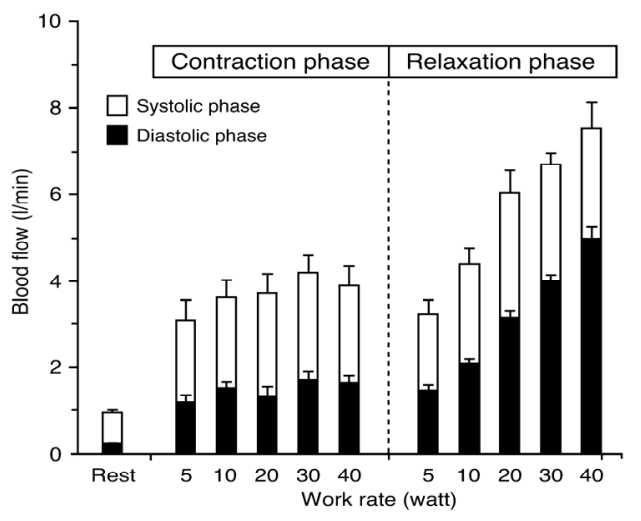

Figure 5. Blood flow at rest between systolic and diastolic phase at muscle contraction and relaxation during 5 - 40 watt exercise at 60 contractions per minute. There is clear increase in blood flow with an increase of work rate during muscle relaxation phase, however there is less change in blood flow between work rates during muscle contraction phase. Values are mean \pm standard error. Figure adapted from Osada and Rådegran [30], reproduced with permission from The Physiological Society of Japan. secutive cycles (1-, 2-, 5-, 10-, 15-, 20- and 30-cycles) for cardiac beat-by-beat cycles at rest, and for muscle contraction-relaxation cycles during steady-state exercise at 10,20, 30 and 40 watt and for 30 and $60 \mathrm{cpm}$, as illustrated by Figure 6. The blood flow variability for seven consecutive cycles was estimated by the coefficients of variation.

In Figure 7, single cardiac beat-by-beat cyclesinduced blood flow variability was almost $15 \%$ at rest. Furthermore, single muscle contraction-relaxation cycleinduced physiological blood flow variability was coefficients of variation, range $11.4 \%-13.2 \%$ at $30 \mathrm{cpm}$, and range $13.3 \%-18.1 \%$ at $60 \mathrm{cpm}$ between 10 and 40 watt. Both at rest and during steady-state exercise, the longer 30-cycles measurements represent a stable condition with minimal blood flow variations (Coefficients of variation, $4.1 \%-4.3 \%$ at $30 \mathrm{cpm}, 4.5 \%-5.8 \%$ at 60 $\mathrm{cpm})$. Furthermore, the 1-muscle contraction-relaxation cycle induced blood flow variability was similar between exercise intensity, but significant variations were seen between contraction frequencies at lower exercise intensities (below 15-cycles at 10 watt and below 5-cycles at
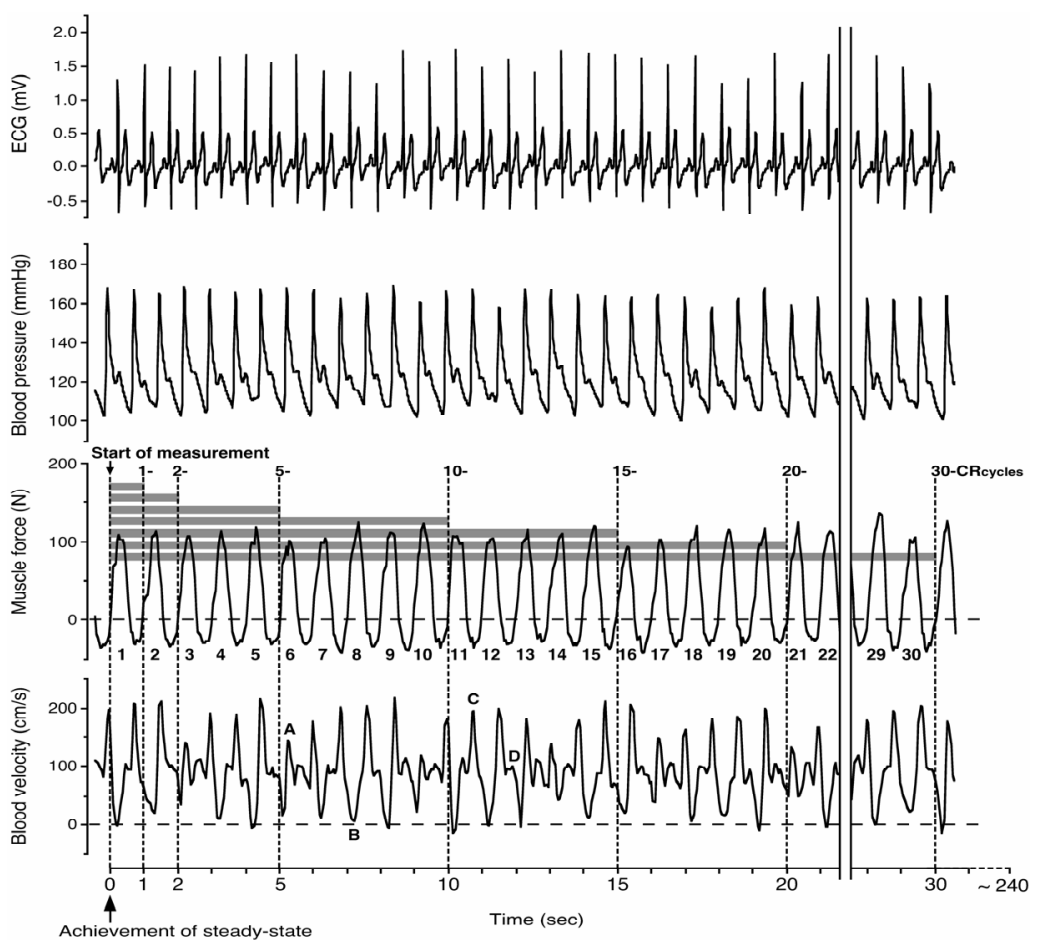

Figure 6. Analysis of blood velocity value for consecutive muscle contraction-relaxation cycles (20 watt and 60 contractions per minute). Blood velocity was measured at steady-state in relation to the muscle force curve for 1-, 2-, 5-, 10-, 15-, 20and 30-consecutive contraction-relaxation cycles $\left(\mathrm{CR}_{\text {cycles }}\right)$. Measurements were guided by the force curve from the initial phase of activity, which represents the initial contraction point ( $\uparrow$ : Start of measurement). The letters depicted indicate; A: Muscle contraction at systolic blood pressure phase, B: Muscle contraction at diastolic blood pressure phase, C: Muscle relaxation at systolic blood pressure phase, D: Muscle relaxation at diastolic blood pressure phase corresponding to Figure 4. Figure adapted from Osada [13], reproduced with permission from Wolters Kluwer Health. 


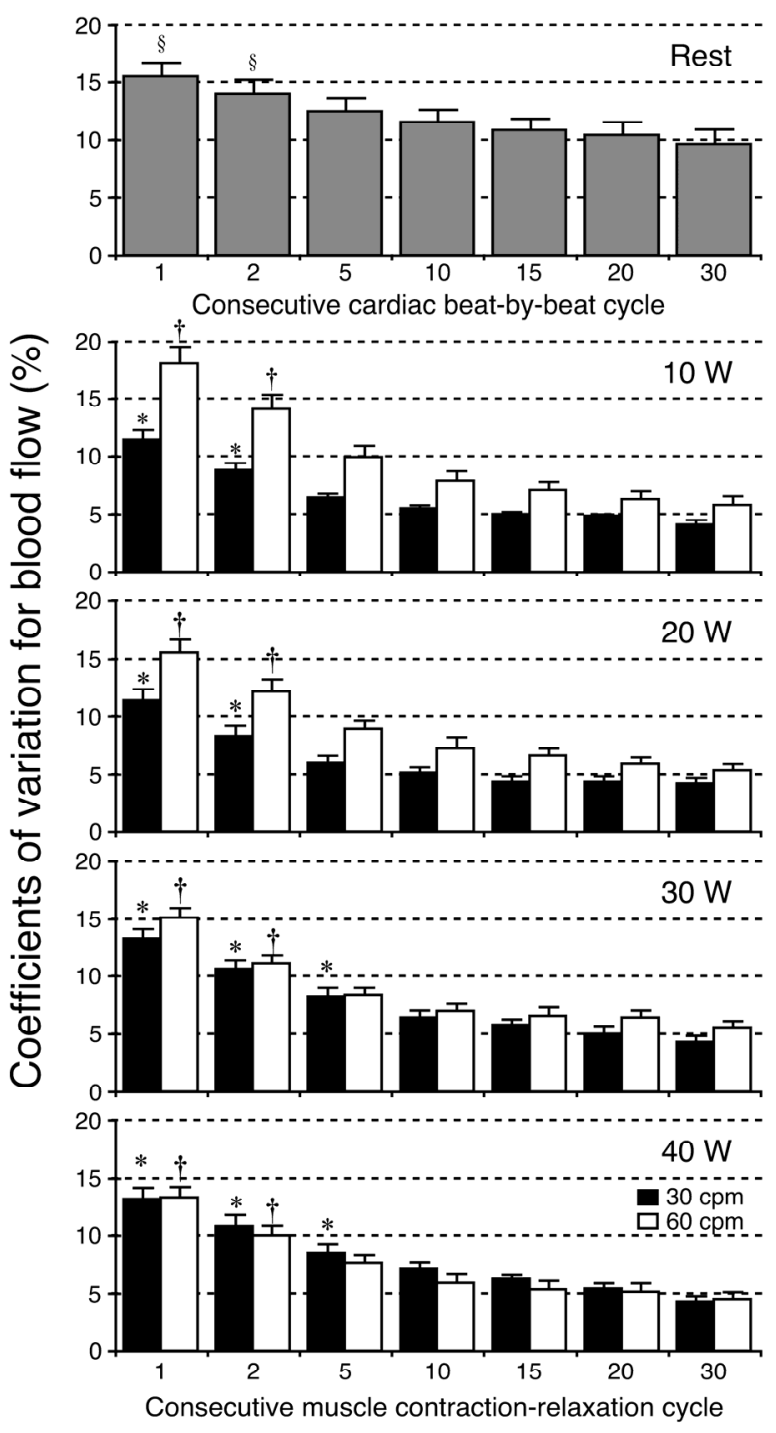

Figure 7. The coefficients of variations for blood flow determined by the consecutive muscle contraction-relaxation cycles $\left(\mathrm{CR}_{\text {cycles }}\right)$. The blood flow variability was determined for seventh consecutive cycles (1-, 2-, 5-, 10-, 15-, 20- and 30 -cycles) for cardiac beat-by-beat cycles at rest, and for $\mathrm{CR}_{\text {cycles }}$ during steady-state exercise at 10, 20, 30 and 40 watt (W) and for 30 and 60 contractions per minute (cpm). The blood flow variability declined exponentially to a stable level; from 1- to 30 - cardiac beat-by-beat cycles at rest, and from 1- to $30-\mathrm{CR}_{\text {cycle }}$ during exercise. The blood flow variability is markedly reduced with a longer sampling measurement of at least $10-\mathrm{CR}_{\text {cycles }}$ with approximately $5 \%$. Values are mean \pm standard error. Figure adapted from Osada [13], reproduced with permission from Wolters Kluwer Health.

beat-by-beat cycle measurement may be closely related to the duration of the beat-by-beat cycle (precisely heart 20 watt. For steady-state rhythmic muscle contractions in the present exercise model, the findings demonstrated that blood flow variability was markedly reduced with a longer sampling measurement of at least 10-muscle contraction-relaxation cycle, which had coefficients of variation of approximately $5 \%$. Above mentioned methodological consideration with samplings number (muscle contraction-relaxation cycle) may be one acceptable procedure for the determination of valid net-blood flow value such as rhythmic and dynamic muscle exercise. However, another exercise model such as intermittent muscle contraction (for instance, the ratio between muscle contraction and relaxation interval is not equal, or both intervals are too long) may cause differences in the magnitude in the muscle contraction-relaxation induced blood flow variability. Though the magnitude in blood flow variability may be different between exercise models, the information regarding its variability and impact on assessment of the steady-state exercising blood flow is available from the non-invasive Doppler method.

\subsection{Comparison in Blood Flow Variability between Muscle Contraction-Relaxation Cycle and Cardiac Beat-by-Beat Cycle}

The resting blood flow evaluated by the beat-by-beat cycle measurements may be a acceptable procedure because of the physiological close relation to magnitude of stroke volume. However, it is still unclear if the evaluation of exercising blood flow should be carried out by muscle contraction-relaxation cycle measurement or the beat-by-beat cycle measurement. The magnitude in netblood velocity determined between muscle contractionrelaxation cycle and beat-by-beat cycle is not similar as illustrated in relation to the blood pressure and force tracings (Figure 3). Therefore, the comparison in blood flow variability (the coefficients of variation) between each beat-by-beat cycle and each muscle contractionrelaxation cycle may provide the more acceptable information for the determination of physiological net-blood flow value during steady-state exercise at different exercise intensities.

Similar blood flow variability between different work rates is seen in muscle contraction-relaxation cycle (Figure 8(A)), however, larger variations in the blood flow with the range of $\sim 18 \%-29 \%$ were observed with an increase in work rate and a shorter duration of the 1beat-by-beat cycle (Figure 8(B)). The duration of the 1muscle contraction-relaxation cycle was, however, constant ( $\approx 1000 \mathrm{~ms}$ corresponding to $60 \mathrm{cpm})$ for the incremental exercise intensities (work rate) resulting in a smaller blood flow variability of approximately $15 \%$ [29].

Such blood flow variability determined for the 1muscle contraction-relaxation cycle measurement has previously also been found to be closely related to the muscle force variability at $60 \mathrm{cpm}$ but not at $30 \mathrm{cpm}$ [13]. On the other hand, the blood flow variability for the 

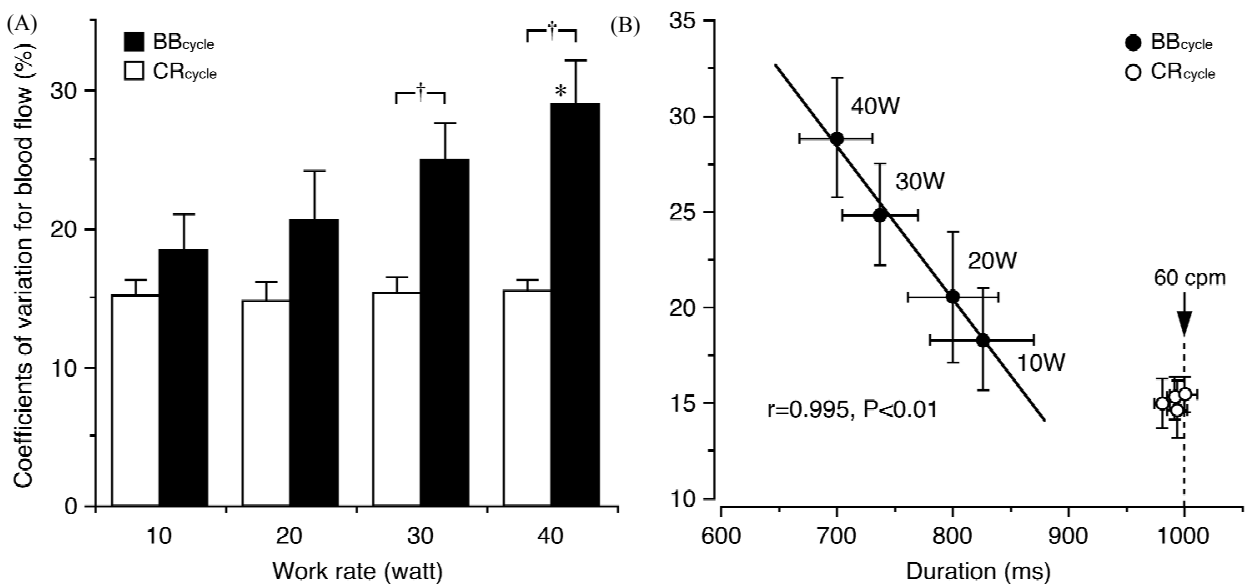

Figure 8. Comparison in blood flow variability between muscle contraction-relaxation cycle $\left(\mathrm{CR}_{\text {cycle }}\right)$ and cardiac beat-by-beat cycle $\left(\mathrm{BB}_{\text {cycle }}\right)$ and its relationship to the duration of a cycle. $(\mathrm{A})$ The coefficients of variation $(\mathrm{CV})$ for blood flow are similar at each work rate when determined for the $\mathrm{CR}_{\text {cycle }}$. The $\mathrm{CV}$ for blood flow determined for the $\mathrm{BB}_{\text {cycle }}$ were, however, significantly ( $^{*} \mathrm{P}<$ $0.05)$ higher at 40 watt $(\mathrm{W})$ compared to at $10 \mathrm{~W}$. A significant $\left({ }^{\dagger} \mathrm{P}<0.01\right)$ difference in the $\mathrm{CV}$ for blood flow was furthermore seen between the $\mathrm{BB}_{\text {cycle }}$ and $\mathrm{CR}_{\text {cycle }}$ at 30 and $40 \mathrm{~W}$, respectively. (B) As the $\mathrm{CV}$ in blood flow were closely $(\mathrm{P}<0.01)$ related to the duration of the $1-\mathrm{BB}_{\text {cycle }}$ but not the $1-\mathrm{CR}_{\text {cycle }}$, the duration of the $1-\mathrm{BB}_{\text {cycle }}$ may be assumed to influence the variations in blood flow. Values are mean \pm standard error. Figure adapted from Osada and Rådegran [29], reproduced with permission from Edizioni Minerva Medica.

rate) with the relative influence of the variations in the blood pressure and intramuscular pressure variations.

\section{CONCLUSION}

The technological development of Doppler ultrasound may contribute to the examination of blood flow dynamics in the exercising muscles in human. The possibility of detection of blood velocity with high temporal resolution in real time at rest as well as during exercise is the advantage for using the Doppler method. However, the enhanced alterations in blood velocity profile may potentially confuse the evaluation of hemodynamics in the exercising muscles. In determining the exercising blood flow measures using non-invasive technique of Doppler ultrasound, the normal physiological variability should be considered when determining the precise physiological net-blood velocity/flow values for the research area in exercise and biomedical science. This review discusses how to obtain accurate measurements of spontaneous changes in exercising blood flow as measured by cardiac beat-by-beat cycle and the muscle contractionrelaxation cycle at various exercise intensities during rhythmmic leg exercise in humans.

\section{ACKNOWLEDGEMENTS}

The staff of The Copenhagen Muscle Research Centre is greatly acknowledged. This study was supported by the Danish National Research Foundation Grant 504-14, Uehara Memorial Foundation in 2002, a Grant-in-Aid for Young Scientists (B) in Scientific Research (No.
16700471) and the "Excellent Young Researchers Overseas Visit Program" in Scientific Research (No. 21-8285) 2010 from MEXT and JSPS.

\section{REFERENCES}

[1] Walløe, L. and Wesche, J. (1988) Time course and magnitude of blood flow changes in the human quadriceps muscles during and following rhythmic exercise. Journal of Physiology (London), 405, 257-273.

[2] Shoemaker, J.K., Hodge, L. and Hughson, R.L. (1994) Cardiorespiratory kinetics and femoral artery blood velocity during dynamic knee extension exercise. Journal of Applied Physiology, 77, 2625-2632.

[3] Rådegran, G. (1997) Ultrasound Doppler estimates of femoral artery blood flow during dynamic knee extensor exercise in humans. Journal of Applied Physiology, 83, 1383-1388.

[4] Osada, T., Katsumura, T., Hamaoka, T., Inoue, S., Esaki, K., Sakamoto, A., Murase, N., Kajiyama, J., Shimomitsu, T. and Iwane, H. (1999) Reduced blood flow in abdominal viscera measured by Doppler ultrasound during onelegged knee extension. Journal of Applied Physiology, 86, 709-719.

[5] Osada, T., Iwane, H., Katsumura, T., Murase, N., Higuchi, H., Sakamoto, A., Hamaoka, T. and Shimomitsu, T. (2012) Relationship between reduced lower abdominal blood flows and heart rate in recovery following cycling exercise. Acta Physiologica, 204, 344-353. doi:10.1111/j.1748-1716.2011.02349.x

[6] Saltin, B., Rådegran, G., Koskolou, M.D. and Roach, R.C. (1998) Skeletal muscle blood flow in humans and its 
regulation during exercise. Acta Physiologica Scandinavica, 162, 421-436. doi:10.1046/j.1365-201X.1998.0293e.x

[7] Sacchetti, M., Saltin, B., Osada, T. and Van Hall, G. (2002) Intramuscular fatty acid metabolism in contracting and non-contracting human skeletal muscle. Journal of Physiology (London), 540, 387-395. doi:10.1113/iphysiol.2001.013912

[8] Steensberg, A., Febbraio, M.A., Osada, T., Schjerling, P., Van Hall, G., Saltin, B. and Pedersen, B.K. (2001) Interleukin-6 production in contracting human skeletal muscle is influenced by pre-exercise muscle glycogen content. Journal of Physiology (London), 537, 633-639. doi:10.1111/j.1469-7793.2001.00633.x

[9] Osada, T. and Rådegran, G. (2002) Femoral artery inflow in relation to external and total work rate at different knee extensor contraction rates. Journal of Applied Physiology, 92, 1325-1330.

[10] Andersen, P. and Saltin, B. (1985) Maximal perfusion of skeletal muscle in man. Journal of Physiology (London), 366, 233-249.

[11] Gill, R.W. (1985) Measurement of blood flow by ultrasound: Accuracy and sources of error. Ultrasound in Medicine and Biology, 11, 625-641. doi:10.1016/0301-5629(85)90035-3

[12] Hughson, R.L., MacDonald, M.J., Shoemaker, J.K. and Borkhoff, C. (1997) Alveolar oxygen uptake and blood flow dynamics in knee extension ergometry. Methods of Information in Medicine, 36, 364-367.

[13] Osada, T. (2004) Muscle contraction-induced limb blood flow variability during dynamic knee extensor. Medicine and Science in Sports and Exercise, 36, 1149-1158. doi:10.1249/01.MSS.0000132272.36832.6A

[14] Rådegran, G. and Saltin, B. (1998) Muscle blood flow at onset of dynamic exercise in humans. American Journal of Physiology Heart and Circulatory Physiology, 274, H314-H322.

[15] Robergs, R.A., Icenogle, M.V., Hudson, T.L. and Greene, E.R. (1997) Temporal inhomogeneity in brachial artery blood flow during forearm exercise. Medicine and Science in Sports and Exercise, 29, 1021-1027. doi:10.1097/00005768-199708000-00006

[16] Isnard, R., Lechat, P., Kalotka, H., Chikr, H., Fitoussi, S., Salloum, J., Golmard, J.-L., Thomas, D. and Komajda, M. (1996) Muscular blood flow response to submaximal leg exercise in normal subjects and in patients with heart failure. Journal of Applied Physiology, 81, 2571-2579.

[17] Leyk, D., Eßfeld, D., Baum, K. and Stegemann, J. (1992) Influence of calf muscle contractions on blood flow parameters measured in the arteria femoralis. International Journal of Sports Medicine, 13, 588-593. doi:10.1055/s-2007-1024571

[18] MacDonald, M.J., Shoemaker, J.K., Tschakovsky, M.E. and Hughson, R.L. (1998) Alveolar oxygen uptake and femoral artery blood flow dynamics in upright and supine leg exercise in humans. Journal of Applied Physiology,
85, 1622-1628.

[19] Sjøgaard, G., Kiens, B., Jørgensen, K. and Saltin, B. (1986) Intramuscular pressure, EMG and blood flow during lowlevel prolonged static contraction in man. Acta Physiologica Scandinavica, 128, 475-484. doi:10.1111/j.1748-1716.1986.tb08002.x

[20] Andersen, P., Adams, R.P., Sjøgaard, G., Thorboe, A. and Saltin, B. (1985) Dynamic knee extension as model for study of isolated exercising muscle in humans. Journal of Applied Physiology, 59, 1647-1653.

[21] Cronestrand, R. (1970) Leg blood flow at rest and during exercise after reconstruction for occlusive disease. Scandinavian Journal of Thoracic Cardiovascular Surgery, 4, $1-24$.

[22] Jorfeldt, L., Juhlin-Dannfelt, A., Pernow, B. and Wassén, E. (1978) Determination of human leg blood flow: A thermodilution technique based on femoral venous bolus injection. Clinical Science and Molecular Medicine, 54, 517-523. doi:10.1016/S0140-6736(64)91518-1

[23] Lassen, N.A., Linbjerg, I. and Munck, O. (1964) Measurement of blood flow through skeletal muscle by intramuscular injection of xenon 133. Lancet, 1, 686-689.

[24] Rådegran, G., Pilegaard, H., Nielsen, J.J. and Bangsbo, J. (1998) Microdialysis ethanol removal reflects probe recovery rather than local blood flow in skeletal muscle. Journal of Applied Physiology, 85, 751-757.

[25] Boushel, R., Langberg, H., Olesen, J., Nowak, M., Simonsen, L., Bülow, J. and Kjær, M. (2000) Regional blood flow during exercise in humans measured by nearinfrared spectroscopy and indocyanine green. Journal of Applied Physiology, 89, 1868-1878.

[26] Ruotsalainen, U., Raitakari, M., Nuutila, P., Oikonen, V., Sipilä, H., Teräs, M., Knuuti, M.J., Bloomfield, P.M. and Iida, H. (1997) Quantitative blood flow measurement of skeletal muscle using oxygen-15-water and PET. Journal of Nuclear Medicine, 38, 314-319.

[27] Jensen, B.R., Sjøgaard, G., Bornmyr, S., Arborelius, M. and Jørgensen, K. (1995) Intramuscular laser-Doppler flowmetry in the supraspinatus muscle during isometric contractions. European Journal of Applied Physiology and Occupational Physiology, 71, 373-378. doi:10.1007/BF00240420

[28] Rådegran, G. (1999) Limb and skeletal muscle blood flow measurements at rest and during exercise in human subjects. Proceedings of Nutrition Society, 58, 887-898. doi:10.1017/S0029665199001196

[29] Osada, T. and Rådegran, G. (2006) Differences in exercising limb blood flow variability between cardiac and muscle contraction cycle related analysis during dynamic knee extensor. Journal of Sports Medicine and Physical Fitness, 46, 590-597.

[30] Osada, T. and Rådegran, G. (2006) Alterations in the blood velocity profile influence the blood flow response during muscle contractions and relaxations. Journal of Physiological Science, 56, 195-203. doi:10.2170/physiolsci.RP002905 